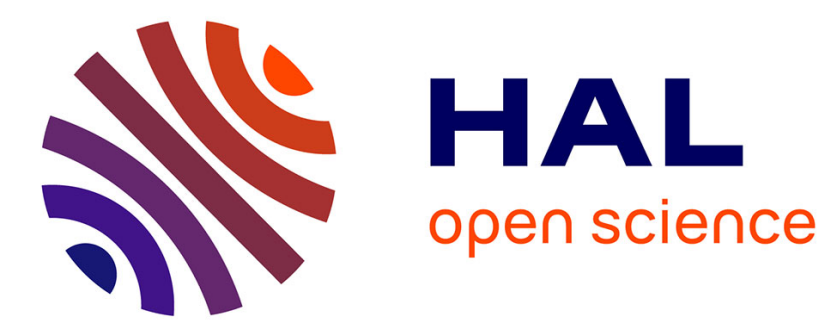

\title{
Aperçu prospectif des déstructurations et restructurations des parcellaires liées à la réalisation de la Ligne Grande VitesseEst européenne
}

Eric Marochini

\section{- To cite this version:}

Eric Marochini. Aperçu prospectif des déstructurations et restructurations des parcellaires liées à la réalisation de la Ligne Grande VitesseEst européenne. Revue Géographique de l'Est, 2002, 42, $10.4000 /$ rge. 2624 . hal-03126164

\section{HAL Id: hal-03126164 \\ https://hal.science/hal-03126164}

Submitted on 30 Jan 2021

HAL is a multi-disciplinary open access archive for the deposit and dissemination of scientific research documents, whether they are published or not. The documents may come from teaching and research institutions in France or abroad, or from public or private research centers.
L'archive ouverte pluridisciplinaire HAL, est destinée au dépôt et à la diffusion de documents scientifiques de niveau recherche, publiés ou non, émanant des établissements d'enseignement et de recherche français ou étrangers, des laboratoires publics ou privés. 


\section{Aperçu prospectif des déstructurations et restructurations des parcellaires liées à la réalisation de la Ligne Grande Vitesse Est européenne}

A Survey of the Dissection and Restructuring of Landholdings caused by the Development of the Eastern European High Speed Rail Link Prospektive Überschau zur Auflösung und Umgestaltung von Parzellen im Hinblick auf die Hochgeschwindigkeits-Linie Ost

\section{Eric Marochini}

\section{OpenEdition Journals}

Édition électronique

URL : http://journals.openedition.org/rge/2624

DOI : 10.4000/rge.2624

ISSN : 2108-6478

\section{Éditeur}

Association des géographes de l'Est

\section{Édition imprimée}

Date de publication : 1 juin 2002

ISSN : 0035-3213

\section{Référence électronique}

Eric Marochini, « Aperçu prospectif des déstructurations et restructurations des parcellaires liées à la réalisation de la Ligne Grande Vitesse

Est européenne », Revue Géographique de l'Est [En ligne], vol. 42 / 3 | 2002, mis en ligne le 14 décembre 2010, consulté le 08 septembre 2020. URL : http://journals.openedition.org/rge/2624 ; DOI : https:// doi.org/10.4000/rge.2624

Ce document a été généré automatiquement le 8 septembre 2020

Tous droits réservés 


\title{
Aperçu prospectif des déstructurations et restructurations des parcellaires liées à la réalisation de la Ligne Grande Vitesse Est européenne
}

\author{
A Survey of the Dissection and Restructuring of Landholdings caused by the \\ Development of the Eastern European High Speed Rail Link \\ Prospektive Überschau zur Auflösung und Umgestaltung von Parzellen im \\ Hinblick auf die Hochgeschwindigkeits-Linie Ost
}

Eric Marochini

\section{Introduction}

1 Après de longues vicissitudes et suite aux enquêtes publiques achevées fin 1994, les travaux de construction d'une ligne nouvelle de chemin de fer à grande vitesse entre Paris et Strasbourg ont été déclarés d'utilité publique (DUP) par un décret du Ministère de l'Equipement, du Logement, des Transports et du Tourisme en date du 14 mai 1996. La réalisation d'un chantier linéaire d'une telle envergure (299 kilomètres de Vairessur-Marne (Seine-et-Marne) à Baudrecourt (Moselle) pour la première tranche) est indubitablement synonyme de bouleversement, de déstructuration des parcellaires agricoles et par voie de conséquence des paysages traversés. Le maître d'ouvrage, à savoir Réseau Ferré de France (RFF), établissement public de l'Etat chargé d'assurer la gestion et le développement du réseau de chemin de fer français, doit légalement veiller à la remise en état des dégâts occasionnés, notamment sur les structures d'exploitation. De même, il doit en même temps limiter, réduire les effets 
environnementaux (impacts hydrauliques, nuisances sonores, barrière et troubles écologiques, etc.) et paysagers.

2 Cet article se propose d'analyser les conséquences de la réalisation de la LGV Est européenne sur les parcellaires agricoles et sur les paysages agraires associés, en dissociant les effets directs liés à la réalisation de l'ouvrage, les réponses qualitatives apportées par les aménageurs de même que les conséquences indirectes induites par les solutions technico-foncières. Les problématiques d'aménagement concernant la traversée du département de la Moselle serviront d'illustration à cette double analyse.

\section{Déstructuration et restructuration des parcellaires agricoles}

3 L'impact sur les structures agricoles de la création de la ligne grande vitesse est fonction d'une multitude de paramètres mais se vit toujours à l'échelle de la commune où le syndrome NIMBY (Not In My Back-Yard - Pas dans mon jardin) est presque toujours vérifiable. Il est notamment plus ou moins important selon la position de la ligne par rapport aux limites et à la forme du finage, selon l'emprise du chantier qui intègre ou non des ouvrages d'art importants (franchissement de route, bassins de rétention, etc.). La déstructuration parcellaire est en fait significativement liée à l'ampleur des travaux et à la ponction foncière à opérer dans la commune traversée. Par ailleurs, les conséquences à attendre en matière de désorganisation des structures parcellaires sont également pleinement conditionnées par les structures foncières initiales et notamment par les éventuelles opérations d'aménagement foncier déjà entreprises sur les bans concernés. De fait, l'impact sera d'autant plus limité que le nombre de parcelles cadastrales, culturales et d'îlots coupés par l'ouvrage sera réduit.

Outre les indemnisations financières obligatoires ayant fait l'objet d'âpres négociations avec les organisations professionnelles agricoles (OPA) (représentant à la fois les propriétaires et les exploitants) et inscrites dans différentes conventions co-signées en 2001 (compensation des pertes de récoltes pour les sondages, pour les fouilles archéologiques, indemnités pour les baisses de rendement des récoltes suivantes, la reconstitution des sols endommagés, le rétablissement des réseaux de drainage, etc.), pour pallier le démantèlement des structures parcellaires, le maître d'ouvrage doit financer des opérations de remembrement agricole quand elles sont nécessaires et souhaitées par les intéressés au plan local. Pour ce faire, dans le cadre de la Ligne Grande Vitesse Est européenne, au regard de la DUP de 1996, les Préfets de département ont saisi les Commissions Départementales d'Aménagement Foncier (CDAF) qui, par arrêté préfectoral, ont indiqué les communes où il y avait lieu, compte tenu des impacts sur le foncier et sur les structures d'exploitation, de créer une Commission Communale d'Aménagement Foncier (CCAF), autorité administrative en charge de la réalisation des remembrements (Ministère de l'Agriculture et de la Pêche, 2001). Avant l'arrêté ordonnant les opérations de remembrement, le maître d'ouvrage, à savoir le Conseil Général par délégation de RFF, fait procéder à une étude d'aménagement (appelée également Pré-étude d'Aménagement Foncier) pour orienter la CCAF dans ses choix d'aménagement au regard d'un diagnostic territorial intégrant les composantes environnementales, paysagères, économiques, foncières et agricoles. En référence à l'article L 123-24 du Code Rural relatif aux remembrements liés à la réalisation d'ouvrages linéaires, deux types de remembrement sont envisageables en 
fonction de l'impact de la ligne et de la structure foncière et agricole traversée : un remembrement avec exclusion ou inclusion de l'emprise.

Dans le premier cas, les terrains constituant l'emprise de l'ouvrage sont exclus du périmètre de remembrement. Ils sont donc acquis par RFF par achat amiable ou par expropriation, en vertu de la DUP, si le propriétaire refuse. A l'intérieur du périmètre perturbé par la réalisation de la ligne, les opérations se déroulent de la même manière qu'un remembrement rural stricto sensu. Le maitre d'ouvrage règle les frais de remembrement et de travaux connexes.

Dans le second cas, la philosophie est tout à fait différente. En effet, le prélèvement de surface nécessaire à la réalisation de la ligne est réparti sur l'ensemble des propriétaires intégrés au périmètre des opérations. Ces prélèvements ne pouvant légalement être supérieurs à $5 \%$, le périmètre doit donc représenter au moins 20 fois la surface de l'emprise. Par exemple, si l'emprise foncière de la ligne dans une commune est de 25 ha, pour pouvoir réaliser un remembrement avec inclusion de cette emprise, le périmètre doit être supérieur ou égal à 500 hectares. Avec ce système, l'association foncière de remembrement devient propriétaire de l'emprise qu'elle rétrocède ensuite au maître d'ouvrage de la ligne. Pour Réseau Ferré de France, ce choix de remembrement est très avantageux. Il peut par ce truchement être autorisé par le Préfet à entrer en possession des terrains avant même que l'arrêté clôturant les opérations de restructuration foncière ne soit signé. Par ailleurs, dans le cas de figure où la Société d'Aménagement Foncier et d'Etablissement Rural (SAFER) possède des terrains dans le périmètre de remembrement, au terme d'un accord entre les deux établissements publics, ces surfaces peuvent être placées sous l'emprise et permettre ainsi de réduire le prélèvement indemnisé sur les autres propriétaires. Le choix d'un remembrement avec inclusion d'emprise permet donc à la CCAF d'envisager une restructuration parcellaire plus globale que dans le premier cas, avec une latitude d'action plus grande. En effet, le remembrement avec exclusion de l'emprise sur une surface minimale, réduite au seul périmètre perturbé par l'ouvrage, limite significativement les possibilités d'échanges entre les propriétaires et n'offre aucunement la possibilité aux municipalités d'envisager la création de réserves foncières pour projeter des aménagements futurs (station de lagunage, lotissement, aire de jeux, etc.).

\section{Des effets paysagers directs et indirects}

7 Bien que consommatrice de très peu d'espace ( 5 à 7 ha au kilomètre), l'insertion d'une ligne nouvelle dans le paysage se traduit par un effet visuel de coupure inéluctable. Nonobstant les efforts qui seront réalisés par les paysagistes pour limiter la lisibilité d'une "cicatrice paysagère » longue de près de 300 kilomètres et les campagnes de communication orchestrées par le maitre d'ouvrage pour faire valoir l'intégration paysagère de la ligne et des ouvrages d'art, les effets paysagers sont incontestables. S'ajoutent à ces incidences directes les effets paysagers induits par les opérations de remembrement réalisées.

8 Aussi, les paysages traversés, perçus depuis la ligne ou depuis le TGV, peuvent-ils connaitre des modifications qui ne remettent certes pas en cause leur structure globale, mais qui peuvent gommer partiellement voire effacer certains repères. Ainsi, pour le " géoagronome " Jean-Pierre Deffontaines (1998), «à grande vitesse, seuls les objets situés à une certaine distance de la voie (au-delà de $400 \mathrm{~m}$ ) sont visibles pendant une 
durée suffisante ». Or, les effets d'une opération de remembrement, notamment dans le cas de figure de l'inclusion d'emprise, vont bien au-delà de cette limite.

Pour panser les plaies paysagères directes, réaliser des effets de résilience, les ingénieurs-paysagistes, au regard des résultats des études paysagères obtenus dans l'avant-projet sommaire (SNCF, 1993) et dans différentes études sectorielles, ont envisagé différents scénarii selon qu'il faille protéger, aménager ou recréer une ambiance paysagère. La politique de "communication paysagère » de RFF vise à un double objectif : rompre l'effet linéaire de l'infrastructure et adapter la ligne aux formes, aux couleurs et à l'ambiance locales. Si le premier objectif peut paraître utopique, des leviers d'actions existent pour le second. Ainsi, à propos de l'insertion des ouvrages d'art (OA), les matériaux et notamment leurs couleurs seront choisis en fonction de l'identité locale. De même, dans l'emprise de la ligne des plantations seront réalisées pour habiller la voie, notamment dans les zones de transition entre les remblais et les déblais. Les délaissés feront également l'objet de reboisements.

Les impacts paysagers des opérations de remembrement sont bien connus, notamment dans les paysages de bocage. Que ce soit directement par l'arasement de haies lors des travaux connexes, par l'entretien trop marqué des cours d'eau, par la définition d'un réseau de chemins simplifié mais aussi plus visible selon les matériaux utilisés, ou indirectement par la nouvelle mise en valeur de l'espace agricole des exploitants après la prise de possession (arasement des haies situées dans de grands îlots, rationalisation et simplification de l'occupation du sol, modification des voies de cheminement), les atteintes paysagères imputables aux remembrements peuvent être importantes mais diversement perceptibles selon l'organisation initiale des paysages concernés (densité et position des haies, lignes créées par le relief, type de structures agraires et d'occupation du sol, etc.) (E. Marochini, 2000). Les dispositions législatives et réglementaires relatives aux remembrements liés à la réalisation d'ouvrages linéaires (ancien article 10 et actuel article L 123-24 du Code Rural), ne différent pas des opérations traditionnelles, sinon que le maitre d'ouvrage doit prendre en charge les frais de travaux connexes à $100 \%$, dans la mesure où ceux-ci concernent une création liée à la nouvelle distribution parcellaire (chemins, réseau hydraulique, etc.) ou à une remise en état, mais en aucun cas à une amélioration de l'existant.

11 La qualité paysagère d'une opération de remembrement est la résultante de nombreuses conditions (E. Marochini, 1999). Comme pour l'ensemble des paramètres environnementaux, ce sont bien les facteurs humains relationnels et la volonté exprimée par la sphère locale qui expliquent les réussites et les échecs en ce domaine. Pourtant, dans le cas des remembrements selon l'article L 123-24, cette volonté de prise en compte, de protection, apparaît quelque peu occultée par le traumatisme occasionné par la réalisation concomitante de la ligne et du remembrement. Pour le corps agricole, toujours fortement représenté dans la CCAF nonobstant la reconnaissance de l'intérêt d'une politique publique en matière de paysages (J.F. Séguin, 2001) et les modifications apportées par la loi paysage de 1993, la déstructuration parcellaire, la modification des itinéraires, la coupure des réseaux d'assainissement et de drainage, placent incontestablement au second plan la question paysagère. Le Ministère de l'Aménagement du Territoire et de l'Environnement, dans une étude récente non publiée, envisageait même que les effets paysagers des opérations de remembrement soient plus marquants que ceux de la ligne elle-même. 


\section{De Cheminot À Baudrecourt (Moselle) : une seconde vague de remembrements aux objectifs strictement agricoles ? (Fig. 1)}

La réalisation de la première tranche de la Ligne Grande Vitesse Est européenne concerne 18 communes mosellanes. De Cheminot à Baudrecourt, les impacts fonciers, agricoles et paysagers de la ligne sont très différents. Dans ce département, la CDAF a décidé de créer des CCAF dans toutes les communes concernées, hormis à Sailly-Achâtel où la position très marginale de l'ouvrage par rapport aux limites du finage ne nécessitait aucun aménagement foncier. Dans ces 17 communes, les pré-études d'aménagement foncier ont été achevées en 2000 et leurs principales conclusions présentées aux CCAF la même année. Au terme de l'enquête publique préalable, 11 communes se sont engagées dans une procédure de remembrement dont deux de manière intercommunale. Comme indiqué sur la figure 1, les communes de Luppy (remembrée depuis peu dans le cadre du contournement de Luppy-Béchy par la RD 910), de Flocourt, de Moncheux, de Thimonville, de Morville-sur-Nied et de Herny ont, quant à elles, choisi de ne pas procéder à un remembrement, compte tenu notamment de l'impact très limité de la ligne sur ces bans communaux.

Figure 1 : Les opérations de remembrement en cours dans le cadre de la réalisation de la LGV Est européenne en Moselle

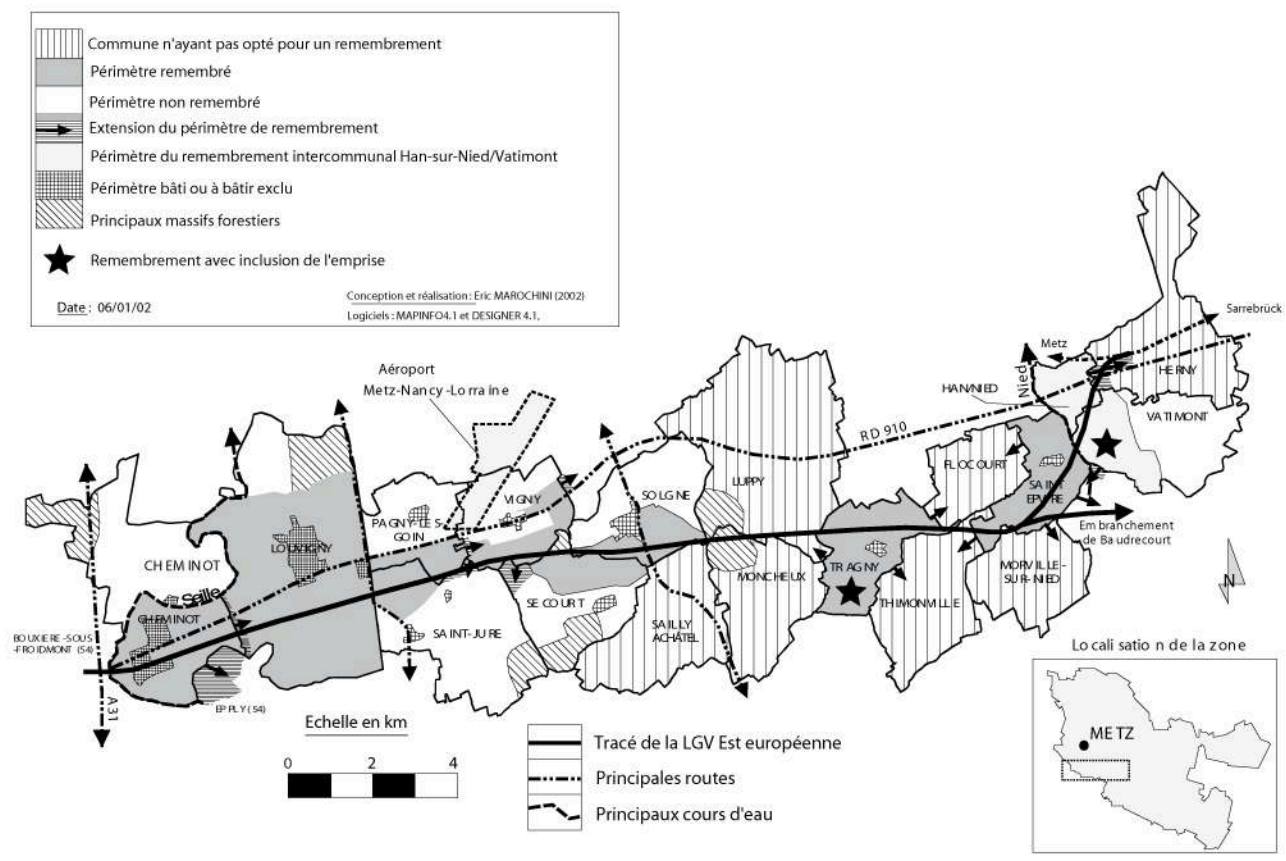

Les destructurations parcellaires ne devraient pas être très marquantes pour ces onze communes. En effet, hormis la commune de Saint-Epvre, l'ensemble des bans a déjà été remembré dans un passé plus ou moins proche. Pour les communes situées à proximité de l'aéroport régional, il peut même s'agir d'un troisième remembrement, dont deux entrepris lors des six dernières années. C'est le cas notamment de la commune de Pagny-lès-Goin, qui vient récemment d'achever les plantations compensatoires dans le cadre du remembrement lié à la réalisation de l'aéroport régional. Cette situation n'est pas sans difficulté. En effet, la faible surface du périmètre (110 ha) limite les possibilités 
d'échanges entre les propriétaires. Bien qu'un nouveau remembrement ait été voté et décidé dans cette commune, au moment du classement des terrains certains exploitants et propriétaires s'interrogeaient déjà sur la réelle opportunité de procéder à cet aménagement. A n'en pas douter, la surface proposée à l'enquête classement devrait d'ailleurs être très différente de celle qui avait été envisagée dans la préétude d'aménagement foncier.

L'influence paysagère des remembrements, notamment dans la partie occidentale du tracé, de Cheminot à Solgne, ne devrait être que très peu sensible. En effet, les paysages $\mathrm{y}$ ont déjà été fortement simplifiés par des pratiques culturales très largement orientées vers des choix de cultures exigeant des structures parcellaires rationalisées (blé, maïs, colza). Dans ces communes, les mesures conservatoires et compensatoires, notamment en matière de haies, devraient d'ailleurs permettre localement d'augmenter les linéaires. Les arrêtés préfectoraux ordonnant ces opérations intègrent d'ailleurs des prescriptions allant en ce sens, mais qui ne s'imposent pas à la CCAF. Il faut donc compter sur le pouvoir de persuasion des aménageurs, notamment le géomètre, le chargé d'études, les Personnes Qualifiées pour la Protection de la Nature (PQPN), et la volonté locale exprimée de s'investir dans une démarche qualité. A cet effet, la Direction Régionale de l'Environnement de Lorraine a organisé en mai 2001 une journée de formation des PQPN à Bonzée-en-Woëvre (Meuse) où les différentes interventions d'agents appartenant aux Directions Départementales de l'Agriculture et de la Forêt des trois départements concernés, de la DIREN, de la SNCF, de RFF et des assistants fonciers ont permis de mettre en place une synergie d'action, une cohérence entre les mesures paysagères prévues dans le cadre de la réalisation de la ligne et celles pouvant être envisagées lors des opérations de remembrement. Dans la partie orientale, à partir de Tragny, les paysages sont beaucoup plus diversifiés, avec une occupation du sol davantage en mosaïque que dans la première partie du linéaire, équilibrée entre terres cultivées et prairies, et où l'arbre et la haie font encore partie de l'ambiance visuelle locale. La question paysagère devra donc y être traitée avec plus d'attention qu'ailleurs.

\section{Conclusion}

La mise en service du TGV est européen est prévue pour la fin de l'année 2006. Avant cette échéance tant attendue en Lorraine, l'ingéniosité et la pertinence des solutions prévues par les aménageurs pour inscrire la ligne avec douceur dans le paysage ne seront certainement pas encore totalement perceptibles et lisibles. A cette même date, les structures d'exploitation auront été réorganisées, restructurées par des opérations de remembrement plus ou moins soucieuses des paysages. Avant même le début des travaux de construction de la ligne et l'achèvement des avant-projets de remembrement, localement, dans les CCAF, la question paysagère n'est pas réellement au centre des débats. L'analyse a posteriori de ces réorganisations des parcellaires montrera les incidences respectives de la cause (LGV) et de la conséquence (remembrement) pour expliquer les éventuelles évolutions paysagères observées. 


\section{BIBLIOGRAPHIE}

DEFFONTAINES J.P. (1998). - Paysages en TGV : regards sur les agricultures, de Paris vers Marseille : fenêtre de droite, Les sentiers d'un géoagronome, Editions Arguments, Paris, 360 p.

Ministère de l'agriculture et de la pêche (2001). - Manuel de procédure de l'aménagement foncier, Paris, $159 \mathrm{p}$.

MAROCHINI E. (1999). - Les remembrements en Moselle entre économie, environnement et société : essai de géographie rurale et appliquée, Thèse de doctorat, Université de Metz, 2 tomes, 601 et 600 p.

MAROCHINI E. (2000). - Aménagement foncier et qualité des paysages : relation possible ou contre nature ?, Action paysagère et acteurs territoriaux, GESTE-MSH Poitiers, Actes de colloque, pp. 187-194.

SEGUIN J.F. (2001). - Politique publique des paysages : entre culture partagée et cohérence territoriale, Le courrier territoires et espaces ruraux, Paris, $n^{\circ} 110$, pp. 4-7.

SNCF (1993). - TGV Est européen : avant-projet sommaire (dossier sectoriel Moselle ouest), 200 p.

\section{RÉSUMÉS}

Cet article se propose d'exposer et d'analyser les différentes solutions utilisables et envisagées par les aménageurs pour limiter les impacts fonciers, agricoles et paysagers dus directement à la réalisation de la Ligne Grande Vitesse Est européenne et à ses conséquences induites.

This article sets out and analyses the different solutions used and considered by the developers for limiting the basic impacts on agriculture and landscapes, caused directly by the construction of the Eastern European High Speed Rail Link, and its indirect consequences.

Dieser Artikel möchte die verschiedenen nutzbaren Lösungen darlegen und analysieren, die ins Auge gefasst wurden, um die Eingriffe in Grundbesitz, Landwirtschaft und Landschaft zu begrenzen, die direkt durch den Bau der Hochgeschwindigkeits-Linie Ost und ihre Folgen entstanden sind.

\section{INDEX}

Mots-clés : département de la Moselle, destructurations, ligne grande vitesse, parcellaires, paysages, restructurations, structures d'exploitation

Schlüsselwörter : Auflösung, Departement Mosel, Hochgeschwindigkeits-Linie, Landschaften, Nutzungs-strukturen, Parzellenmuster, Umgestaltung

Keywords : Department of Moselle, dissection, High Speed Rail Link, landholdings, landscapes, operating structures, restructuring 
AUTEUR

\section{ERIC MAROCHINI}

Mairie de Fameck - 29, avenue Jeanne-d'Arc 57290 FAMECK - email :

sm.mairiefameck@wanadoo.fr 